Check for updates

Cite this: RSC Adv., 2019, 9, 37082

\section{A selective and sensitive UFLC-MS/MS method for the simultaneous determination of five alkaloids from Piper longum L. and its application in the pharmacokinetic study of 6-OHDA-induced Parkinson's disease rats}

\author{
Rongrong Xu, D Wenwen Zhao, Lan Yu, Qijun Chen, Xiaolu Hu, Yinying Ba, \\ Xiaoqing Chen, (D) Xing Wang and Xia Wu*
}

\begin{abstract}
The alkaloids from Piper longum L. (PLA) mainly contain piperine (PPR), piperlongumine (PPL), $\Delta \alpha, \beta$ dihydropiperlonguminine (DPPL), piperanine (PPRA) and pellitorine (PLTR), which have neuroprotective effects on a 6-OHDA-induced rat model of Parkinson's disease (PD). To elucidate the pharmacokinetic profiles of these main compounds in PD rats, we developed a rapid, selective and sensitive ultra-fast liquid chromatography-electronic spray ionization-tandem mass spectrometry (UFLC-ESI-MS/MS) method which was validated for the simultaneous determination of the 5 absorbed compounds in the plasma of 6-OHDA-induced PD rats. The plasma samples were pretreated using a protein precipitation method with methanol/acetonitrile $(1: 1, \mathrm{v} / \mathrm{v})$. The analytes and internal standard (IS) were separated on a Phenomenex Gemini C18 column using gradient elution with a mobile phase consisting of acetonitrile and a $0.1 \%$ formic acid aqueous solution at a flow rate of $0.5 \mathrm{~mL} \mathrm{~min}{ }^{-1}$. The total chromatographic running time was $4.5 \mathrm{~min}$. The detection was performed with positive electrospray ionization (ESI) using the multiple reaction monitoring (MRM) mode of transitions at $\mathrm{m} / \mathrm{z} 286.2 \rightarrow 201.2, \mathrm{~m} / \mathrm{z} 274.2 \rightarrow 201.2$, $\mathrm{m} / \mathrm{z} 276.2 \rightarrow 135.1, \mathrm{~m} / \mathrm{z} 288.2 \rightarrow 135.1, \mathrm{~m} / \mathrm{z} 224.1 \rightarrow 168.2$, and $\mathrm{m} / \mathrm{z} 472.1 \rightarrow 436.1$ for PPR, PPL, DPPL, PPRA, PLTR and IS, respectively. All five analytes showed excellent linearity $(R>0.995)$ within the concentration range of $0.20-5000 \mathrm{ng} \mathrm{mL}^{-1}$. The established method was then applied to investigate the pharmacokinetics of multi-components (PPR, PPL, DPPL, PPRA and PLTR) in PD rats after oral administration of PLA. The results showed that no obvious differences were observed in the pharmacokinetic parameters of PPR, PPL, DPPL, PPRA and PLTR in PD rats compared with those in sham rats after oral administration of PLA except for MRTs for PPR, PPL and PLTR. Additionally, the activities of superoxide dismutase (SOD) were related to the concentrations of the multi-components in plasma.
\end{abstract}

Received 14th August 2019 Accepted 27th October 2019

DOI: 10.1039/c9ra06325k

rsc.li/rsc-advances pellitorine (PLTR), exerted neuroprotective effects in Parkinson's disease (PD) models induced by neurotoxins such as 6hydroxydopamin (6-OHDA), 1-methyl-4-phenyl-1,2,3,6tetrahydropyridine (MPTP), rotenone and lipopolysaccharide (LPS). Furthermore, PPR and PPL could improve cell viability and alleviate motor deficits and loss of midbrain dopaminergic neurons in PD cell and mouse models induced by rotenone. These effects had an association with restoring the balance between autophagy and apoptosis. It was also found that the activity of superoxide dismutase (SOD) was enhanced in PD rat after administration of PLA. ${ }^{4-9}$ SOD plays a significant role in protecting cells from oxidative stress and preventing neuronal apoptosis.

The pharmacokinetic behaviors of the components in the model group might be different compared with those in the normal group due to the changes of the function and expression of many enzymes, transporters and intestinal bacteria related to
Beijing Key Lab of TCM Collateral Disease Theory Research, School of Traditional Chinese Medicine, Capital Medical University, 10 Xitoutiao, Youanmen, Beijing, China. E-mail: wuxia6710@163.com; Fax: +86-10-83911627; Tel: +86-10-83911671 
the transportation and metabolism of drugs in the pathological status. ${ }^{10-12}$ A few studies have reported pharmacokinetics of traditional Chinese medicine in the models of neurological diseases such as Alzheimer's disease (AD), chronic depression, insomnia and migraine. The results indicated that most of components had no obvious differences in pharmacokinetic parameters between the normal and model group, while only the absorption of some compounds were changed more rapidly in model group than those in the normal group. ${ }^{13-16}$ The pharmacokinetic evaluations of some drugs and pro-drugs for treatment of patients with PD by stabilizing levodopa plasma concentration have been reported recently. ${ }^{\mathbf{1 7 - 2 0}}$ But few studies on the pharmacokinetics of traditional Chinese medicine in the animal models of PD were reported by now except for Shouwu Fang. ${ }^{21}$ Our previous research focused on the pharmacokinetic properties of PPR and PPL and tissue distribution characteristics of PPR, PPL and DPPL in normal rats after oral administration of PLA, which showed that these three alkaloids could cross the blood-brain barrier. ${ }^{22,23}$ Based on the above considerations, we would like to know if there are differences in the pharmacokinetic characteristics of the main alkaloids of PLA between the PD rats and the normal rats.

The aims of the study were to develop a rapid, accurate, selective and sensitive ultra-fast liquid chromatographyelectronic spray ionization-tandem mass spectrometry (UFLCESI-MS/MS) method for determining simultaneously the contents of 5 amide alkaloids (PPR, PPL, DPPL, PPRA and PLTR) in rat plasma, and then applied to comparative pharmacokinetics of multi-components in the 6-OHDA-induced PD rats and the sham rats after oral administration of PLA. The correlations between the SOD activities and concentrations of five alkaloids in plasma were investigated. The results could provide some evidences for understanding the mechanism of PLA in dynamic perspective and give valuable information for drug development as an antiparkinsonian agent.

\section{Materials and methods}

\section{Chemicals and regents}

PLA was prepared by our laboratory and contained $49.07 \%$ PPR, $1.42 \%$ PPL, $0.62 \%$ DPPL, $2.60 \%$ PPRA and $4.82 \%$ PLTR, which were determined by UFLC-ESI-MS/MS. P. longum was extracted with $75 \%$ ethanol by reflux extraction, fractionated by D101 macroporous resin column chromatography and eluted with the gradient ethanol- $\mathrm{H}_{2} \mathrm{O}(20: 80,60: 40,85: 15, \mathrm{v} / \mathrm{v})$ to give three fractions (Frs I-III). Fr. II was evaporated to dryness, which was used as PLA. Terfenadine (internal standard, IS) were purchased from Sigma-Aldrich (St. Louis, MO, USA). The reference standards of PPR, PPL, DPPL, PPRA and PLTR were isolated and purified by our laboratory. Their structures shown in Fig. 1 were identified by Fourier transform mass spectrometry (FT-MS) and nuclear magnetic resonance (NMR). The purities of all the reference standards determined by HPLC are higher than 98\%. SOD kit was bought from Nanjing Jiancheng Bioengineering Institute (Jiangsu, China).

LC-MS grade methanol, acetonitrile and HPLC-grade formic acid were purchased from Fisher (Fair Lawn, NJ, USA). HPLC- grade water was prepared using a Milli-Q water purification system (Millipore, MA, USA).

\section{Instruments and conditions}

Assays were carried out on an LC-20A series UFLC system (Shimadzu, Kyoto, Japan) equipped with a system controller (CBM-20A), a binary solvent delivery system (LC-20AD), an automatic injector (SIL-20AC), a column oven (CTO-20A) and an API 4000 triple-quadrupole mass spectrometer coupled with an electrospray ionization (ESI) source (Applied Biosystems, CA, USA). Analyst software (version 1.6.1) was used for data acquisition and processing. A Phenomenex Gemini C18 column $(50 \mathrm{~mm} \times 2.00 \mathrm{~mm}, 5 \mu \mathrm{m})$ was used to carry out the chromatographic separation of the analytes and IS. The mobile phase system was composed of $0.1 \%$ formic acid aqueous solution (A) and acetonitrile (B). The optimized UFLC elution conditions were: $0.01-0.60 \mathrm{~min}, 10 \% \mathrm{~B}, 0.60-1.50 \mathrm{~min}, 10-98 \%$ B, $1.50-3.00 \mathrm{~min}, 98 \% \mathrm{~B}, 3.00-3.01 \mathrm{~min}, 98-10 \% \mathrm{~B}, 3.01-$ $4.50 \mathrm{~min}, 10 \% \mathrm{~B}$. The flow rate was set at $0.5 \mathrm{~mL} \mathrm{~min}^{-1}$ and the injection volume was $10 \mu \mathrm{L}$. The column was maintained at room temperature.

The MS analyses were operated in positive ESI mode, and the data were acquired by multiple reaction monitoring (MRM). High purity nitrogen was used as the curtain gas (CUR), nebulizer gas (GS1) and heater gas (GS2). The optimized parameters were as follows: pressure of CUR, 20 psi; pressure of GS1 and GS2, $60 \mathrm{psi}$; ion spray voltage, $5000 \mathrm{~V}$; capillary temperature, $550{ }^{\circ} \mathrm{C}$; entrance potential (EP), $10 \mathrm{~V}$; collision-activated dissociation (CAD), medium; collision cell exit potential (CXP), $15 \mathrm{~V}$. The selected precursor ion of Q1 $(\mathrm{m} / \mathrm{z})$ and product ion of Q3 $(\mathrm{m} /$ $z$ ), declustering potential (DP) and collision energy (CE) of PPR, PPL, DPPL, PPRA, PLTR and IS are listed in Table 1.

\section{Preparation of standard solutions}

PPR, PPL, DPPL, PPRA and PLTR was dissolved in methanol and prepared to $1.00 \mathrm{mg} \mathrm{mL} \mathrm{mL}^{-1}$ stock standard solutions. Standard solutions, which contained eight concentrations of 2, 5, 20, 50, 200, 500, 1000 and $2000 \mathrm{ng} \mathrm{mL}{ }^{-1}$ for PPR, PPL, DPPL and PPRA and 50, 125, 500, 1250, 5000, 12 500, 25000 and $50000 \mathrm{ng} \mathrm{mL}$ for PLTR, and quality control (QC) solutions at low, medium and high concentration $\left(6,100,1600 \mathrm{ng} \mathrm{mL}^{-1}\right.$ for PPR, PPL, DPPL and PPRA; 150, 2500, $40000 \mathrm{ng} \mathrm{mL}^{-1}$ for PLTR) were prepared by further serially diluting the stock solution with methanol. The IS stock solution $\left(1 \mathrm{mg} \mathrm{mL}^{-1}\right)$ was also prepared in methanol and then diluted to a final concentration of $10 \mathrm{ng}$ $\mathrm{mL}^{-1}$ with methanol/acetonitrile $(1: 1, \mathrm{v} / \mathrm{v})$.

\section{Sample preparation}

A $50 \mu \mathrm{L}$ aliquot of plasma sample mixed with $300 \mu \mathrm{L}$ of IS solution and $5 \mu \mathrm{L}$ methanol (or a standard or QC solution) was vortexed for $3 \mathrm{~min}$, and then centrifuged at $12000 \mathrm{~g}$ for $10 \mathrm{~min}$. The supernatant was separated and stored, of which $10 \mu \mathrm{L}$ was injected for analysis. Prior to analysis, samples with concentrations above the highest standard were diluted with blank plasma. 

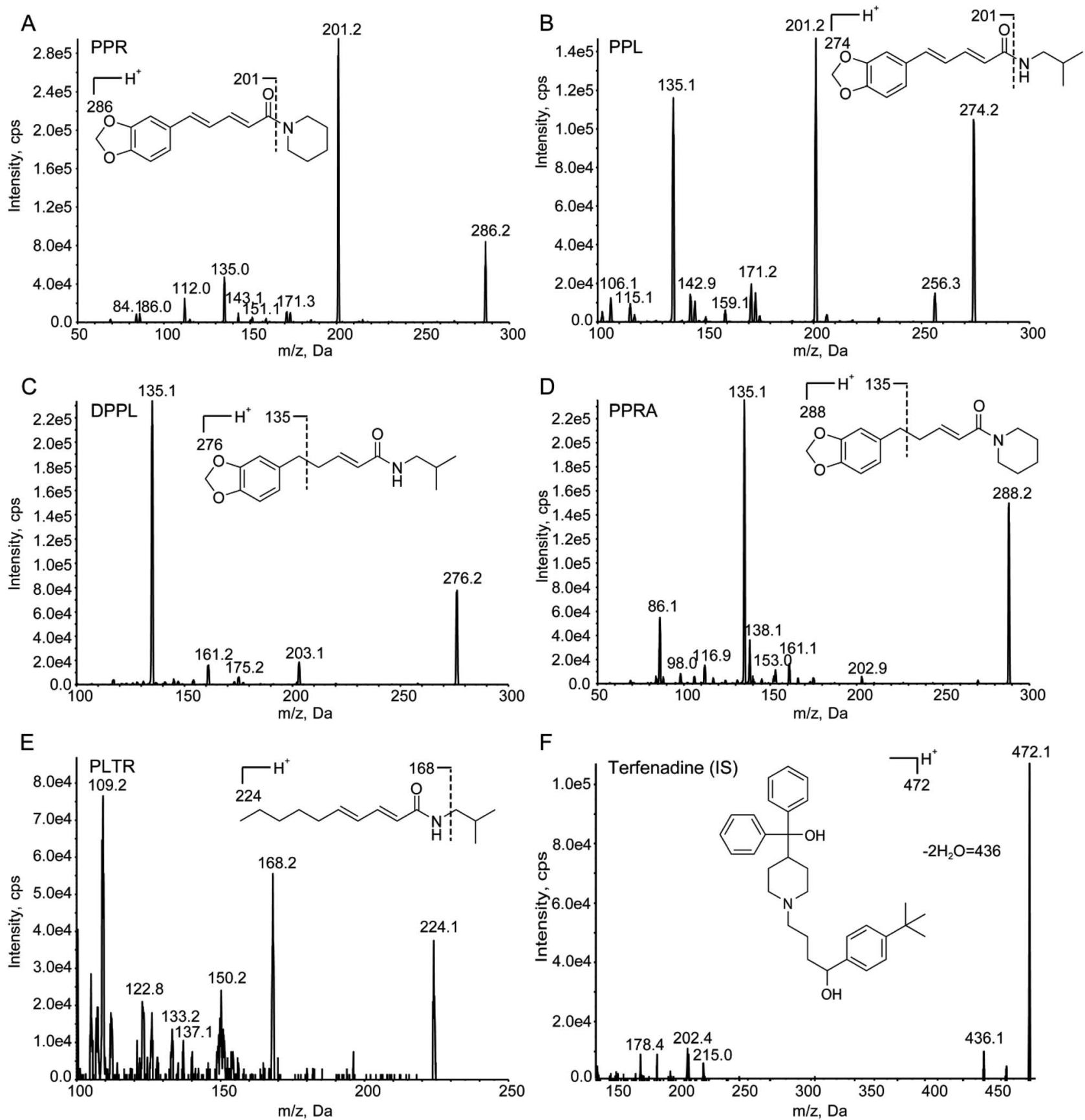

Fig. 1 Full-scan product ion spectra of $[M+H]^{+}$ions and fragmentation pathways for (A) PPR, (B) PPL, (C) DPPL, (D) PPRA, (E) PLTR and (F) terfenadine (IS)

Table 1 The precursor ion, product ion, DP and CE of PPR, PPL, DPPL, PPRA, PLTR and IS

\begin{tabular}{lllll}
\hline Compound & Q1 $(m / z)$ & Q3 $(m / z)$ & DP $(\mathrm{V})$ & CE $(\mathrm{eV})$ \\
\hline PPR & 286.2 & 201.2 & 112 & 28 \\
PPL & 274.2 & 201.2 & 94 & 25 \\
DPPL & 276.2 & 135.1 & 97 & 32 \\
PPRA & 288.2 & 135.1 & 117 & 38 \\
PLTR & 224.1 & 168.2 & 93 & 27 \\
IS & 472.1 & 436.1 & 130 & 50
\end{tabular}

\section{Method validation}

Selectivity. The chromatograms of blank plasma samples, blank plasma samples spiked with standards and IS, and plasma samples collected after oral administration of PLA spiked with IS were compared and evaluated the selectivity of the method.

Linearity of calibration and LLOQs. The calibration curves for the five alkaloids were established respectively by plotting peak area ratio $(y)$ of each analyte to IS versus concentration of each analyte $(x)$ using the weighted $\left(1 / x^{2}\right)$ least-square linear 
regression. Lower limit of quantification (LLOQ) was defined as the lowest concentration on each calibration curve of corresponding analyte which was determined with acceptable precision and accuracy within $20 \%$ at the signal to noise $(\mathrm{S} / \mathrm{N})$ ratio of $10: 1$.

Precision and accuracy. Six replicate QC samples at three concentration levels (low, medium, and high) were analyzed on the same day and on three consecutive days for the intra/interday precision and accuracy, respectively. The precision was expressed as relative standard deviation (RSD, \%) and accuracy as relative error (RE, \%).

Extraction recovery and matrix effect. The extraction recoveries of five compounds were evaluated by comparing the analytical results of extracted QC samples with those of postextracted blank plasma samples spiked with the analytes and
IS at corresponding concentrations. The matrix effect was assessed by comparing the analytical results of samples obtained by spiking the analytes and IS into post-extracted matrix with those of samples obtained by spiking the analytes and IS into methanol.

Stability. The stabilities of five analytes in rat plasma were evaluated with QC samples at three concentration levels under various storage and processing conditions including three freeze-thaw cycles, long-term cryopreservation $\left(-80{ }^{\circ} \mathrm{C}, 30 \mathrm{~d}\right)$, short-term placement $\left(4^{\circ} \mathrm{C}, 48 \mathrm{~h}\right)$ and autosampler $\left(20^{\circ} \mathrm{C}, 4 \mathrm{~h}\right)$.

\section{Animals}

Fifteen male Sprague-Dawley rats $(220 \pm 20 \mathrm{~g})$ were purchased from the Beijing Vital River Lab Animal Technology Co., Ltd. (Beijing, China). The rats were housed under standard
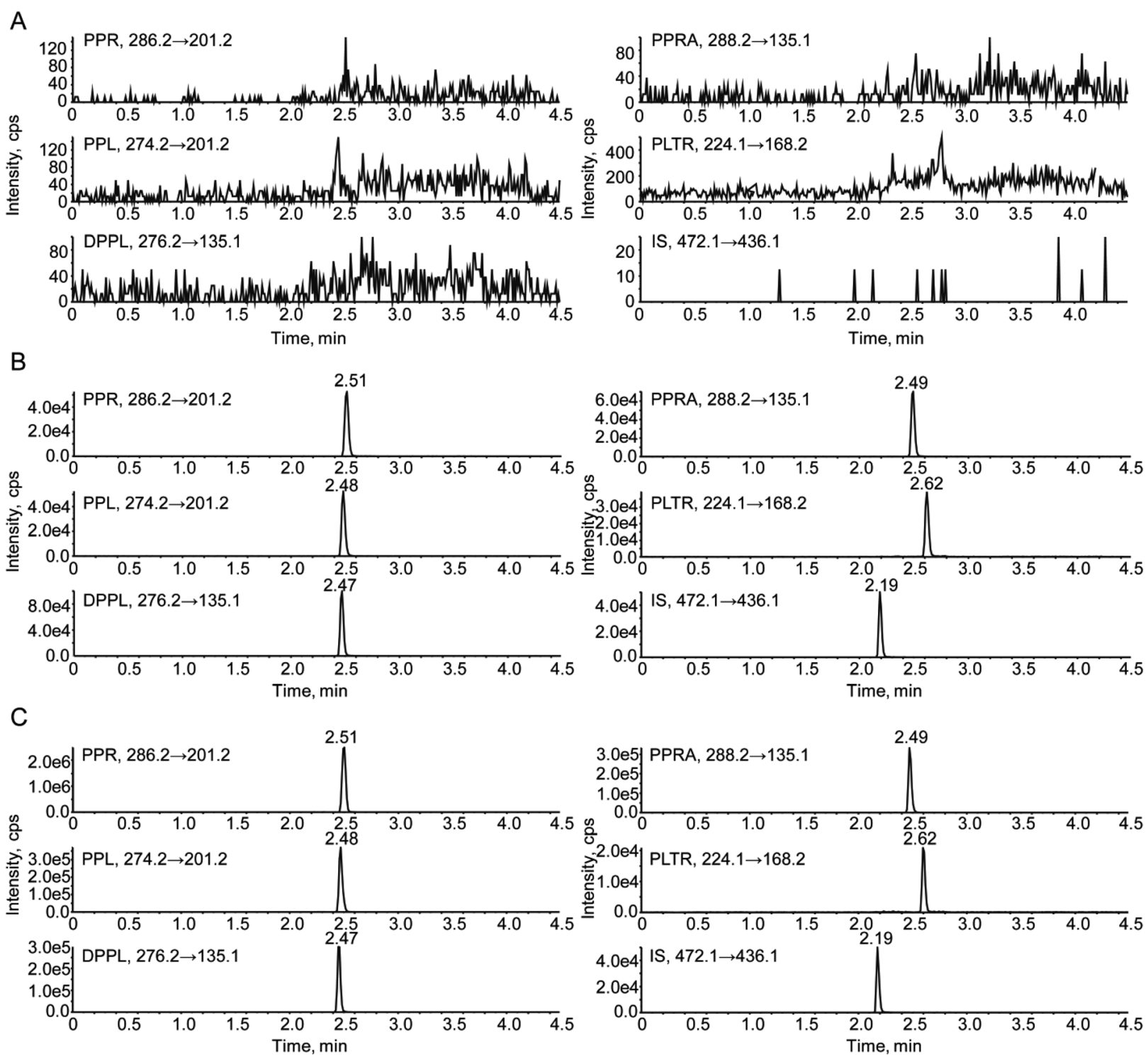

Fig. 2 Representative chromatograms of (A) blank rat plasma, (B) blank rat plasma spiked with PPR (10 $\left.\left.\mathrm{ng} \mathrm{mL}^{-1}\right), \mathrm{PPL}^{(10 \mathrm{ng} \mathrm{mL}}{ }^{-1}\right), \mathrm{DPPL}(10 \mathrm{ng}$ $\left.\mathrm{mL}^{-1}\right)$, PPRA $\left(10 \mathrm{ng} \mathrm{mL}^{-1}\right), \operatorname{PLTR}\left(250 \mathrm{ng} \mathrm{mL}^{-1}\right)$ and IS $\left(1.0 \mathrm{ng} \mathrm{mL}^{-1}\right)$, and (C) an unknown PD rat plasma sample collected at $45 \mathrm{~min}$ after oral administration of $50 \mathrm{mg} \mathrm{kg}^{-1}$ PLA. 
Table 2 Regression equations, correlation coefficients, linearity ranges and LLOQs of PPR, PPL, DPPL, PPRA and PLTR in rat plasma

\begin{tabular}{|c|c|c|c|c|}
\hline Compound & $\begin{array}{l}\text { Range (ng } \\
\mathrm{mL}^{-1} \text { ) }\end{array}$ & Calibration curves & $\begin{array}{l}\text { Correlation coefficient } \\
(R)\end{array}$ & LLOQ (ng mL $\left.\mathrm{m}^{-1}\right)$ \\
\hline PPR & $0.2-200$ & $y=0.1060 x+0.00605$ & 0.9978 & 0.2 \\
\hline PPL & $0.2-200$ & $y=0.0978 x+0.00422$ & 0.9975 & 0.2 \\
\hline DPPL & $0.2-200$ & $y=0.1640 x+0.00866$ & 0.9980 & 0.2 \\
\hline PPRA & $0.2-200$ & $y=0.1270 x+0.00627$ & 0.9971 & 0.2 \\
\hline PLTR & $5-5000$ & $y=0.0027 x-0.00088$ & 0.9978 & 5.0 \\
\hline
\end{tabular}

conditions at a temperature of $25 \pm 2{ }^{\circ} \mathrm{C}$, a relative humidity of $50 \pm 15 \%$, a $12 \mathrm{~h}$ dark-light cycle, with food and water were provided ad libitum. All experimental procedures were approved by Animal Experiments and Experimental Animal Welfare Committee of Capital Medical University. Our research had acquired the ethics approval with the number AEEI-2015-082.

Table 3 Precisions, accuracies, recoveries and matrix effects of PPR, PPL, DPPL, PPRA and PLTR in QC samples at three concentration levels ( $n=$ 6)

\begin{tabular}{|c|c|c|c|c|c|c|c|}
\hline \multirow[b]{2}{*}{ Compound } & \multirow[b]{2}{*}{$\begin{array}{l}\text { Spiked (ng } \\
\mathrm{mL}^{-1} \text { ) }\end{array}$} & \multicolumn{2}{|l|}{ Intra-day } & \multicolumn{2}{|l|}{ Inter-day } & \multirow[b]{2}{*}{$\begin{array}{l}\text { Recovery }(\%, \\
\text { mean } \pm \mathrm{SD})\end{array}$} & \multirow{2}{*}{$\begin{array}{l}\text { Matrix effect (\%, } \\
\text { mean } \pm \\
\text { SD) }\end{array}$} \\
\hline & & $\begin{array}{l}\text { Precision (RSD, } \\
\%)\end{array}$ & $\begin{array}{l}\text { Accuracy (RE, } \\
\%)\end{array}$ & $\begin{array}{l}\text { Precision (RSD, } \\
\%)\end{array}$ & $\begin{array}{l}\text { Accuracy (RE, } \\
\%)\end{array}$ & & \\
\hline \multirow[t]{3}{*}{ PPR } & 0.6 & 7.71 & 0.83 & 2.84 & -1.05 & $90.75 \pm 6.42$ & $101.60 \pm 6.45$ \\
\hline & 10 & 7.75 & -6.78 & 2.51 & -4.16 & $94.44 \pm 1.87$ & $98.84 \pm 1.91$ \\
\hline & 160 & 2.69 & -11.88 & 3.78 & -8.65 & $98.17 \pm 0.93$ & $98.92 \pm 1.39$ \\
\hline \multirow[t]{3}{*}{ PPL } & 0.6 & 2.51 & 5.94 & 5.24 & 2.30 & $81.10 \pm 7.45$ & $104.52 \pm 8.45$ \\
\hline & 10 & 3.97 & 3.68 & 4.03 & 2.33 & $94.11 \pm 1.95$ & $99.66 \pm 2.12$ \\
\hline & 160 & 4.40 & -5.10 & 5.05 & -3.89 & $99.75 \pm 1.60$ & $99.03 \pm 2.52$ \\
\hline \multirow[t]{3}{*}{ DPPL } & 0.6 & 2.94 & 1.03 & 5.10 & -0.64 & $85.03 \pm 2.95$ & $96.96 \pm 6.69$ \\
\hline & 10 & 10.8 & 8.00 & 4.25 & 5.22 & $97.00 \pm 1.32$ & $99.92 \pm 2.12$ \\
\hline & 160 & 4.69 & -2.60 & 4.11 & -3.65 & $100.67 \pm 1.78$ & $99.02 \pm 1.97$ \\
\hline \multirow[t]{3}{*}{ PPRA } & 0.6 & 4.35 & -1.36 & 0.56 & -0.72 & $94.37 \pm 3.99$ & $100.76 \pm 4.96$ \\
\hline & 10 & 5.03 & 3.07 & 2.87 & 1.27 & $97.67 \pm 1.17$ & $99.39 \pm 2.82$ \\
\hline & 160 & 3.19 & -6.67 & 3.17 & -5.66 & $100.33 \pm 1.47$ & $100.70 \pm 2.77$ \\
\hline \multirow[t]{3}{*}{ PLTR } & 15 & 6.69 & 3.00 & 4.65 & -1.22 & $88.92 \pm 3.02$ & $90.63 \pm 6.96$ \\
\hline & 250 & 4.15 & 3.87 & 6.65 & -3.53 & $103.72 \pm 2.86$ & $99.07 \pm 4.21$ \\
\hline & 4000 & 1.97 & 4.46 & 4.54 & -0.04 & $102.57 \pm 1.53$ & $98.70 \pm 2.19$ \\
\hline
\end{tabular}

Table 4 Stability of PPR, PPL, DPPL, PPRA and PLTR under different conditions $(n=3)$

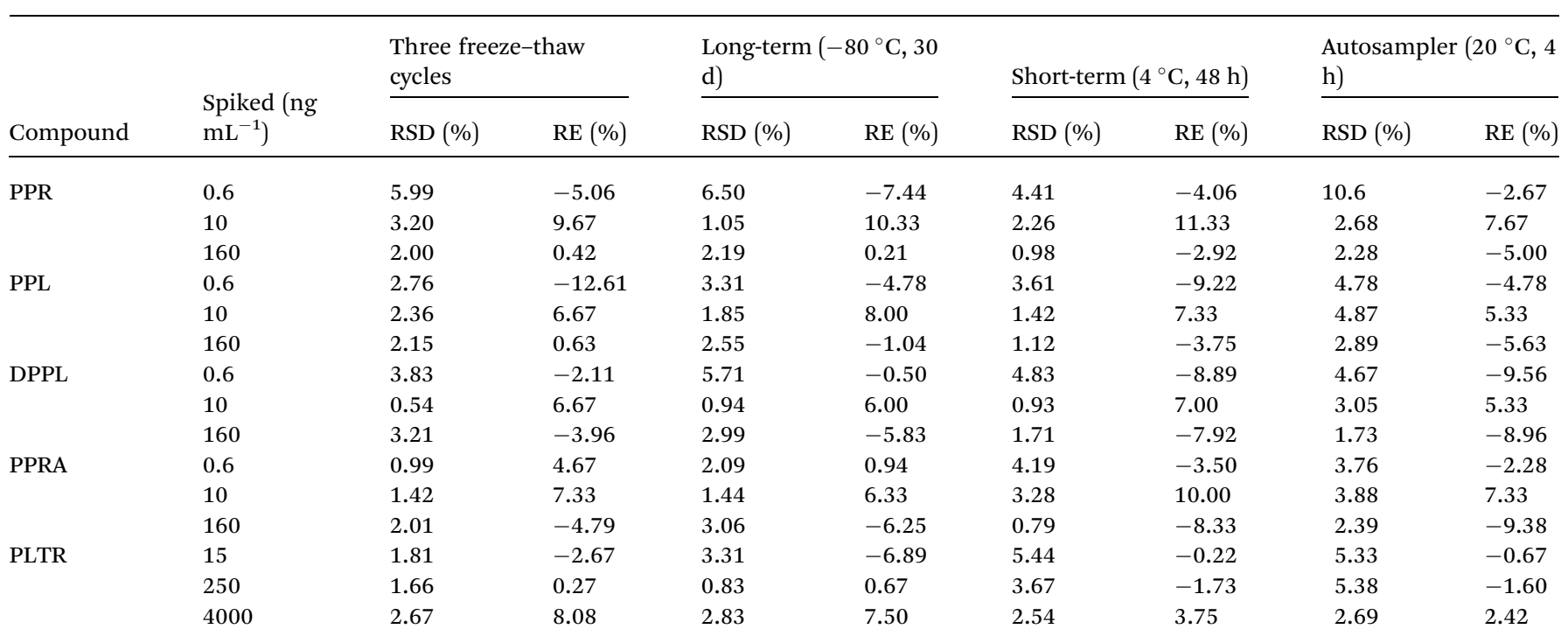


After one week of acclimatization to the new surroundings before experimental surgery, the rats were randomly divided into two groups: sham group $(n=5)$, PD group $(n=10)$. The PD rats were injected $3.0 \mu \mathrm{L}$ 6-OHDA $\left(4 \mathrm{mg} \mathrm{mL}^{-1}\right)$ which was dissolved in $0.02 \%$ ascorbic acid saline into the left striatum following a previous described protocol. ${ }^{6}$ The three injection positions were anteroposterior $0.7 \mathrm{~mm}$, lateral $2.6 \mathrm{~mm}$ and dorsoventral 6.0, 5.5, $5.0 \mathrm{~mm}$ from bregma, respectively. Inject $1.0 \mu \mathrm{L} 6$-OHDA solution gradually into each target point at a rate of $1.0 \mu \mathrm{L} \mathrm{min}{ }^{-1}$. After injection, the needle was left in the target area for $5 \mathrm{~min}$ to prevent backfilling and then slowly removed. The sham group was injected with $1.0 \mu \mathrm{L}$ vehicle $(0.02 \%$ ascorbic acid saline). After 5 weeks of the surgery, the rats were injected subcutaneously with $0.5 \mathrm{mg} \mathrm{kg}^{-1}$ apomorphine which was dissolved in physiological saline to observe the rotational behavior. Two days before the experiment, the jugular veins of anesthetized rats injected intravenously with $50 \mathrm{mg} \mathrm{kg} \mathrm{kg}^{-1}$ pentobarbital were cannulated with polyethylene tubing. The cannula filled with heparinized saline (20 units per $\mathrm{mL}$ ) was externalized at the back of the neck.

\section{Application in pharmacokinetic study}

The sham rats were defined as PLA-Sham group. The PD rats were divided into PLA-PD group and PPR-PD group. Both PLASham and PLA-PD groups were orally administered a $50 \mathrm{mg}$ $\mathrm{kg}^{-1}$ dose of PLA, and PPR-PD group was orally administered a $25 \mathrm{mg} \mathrm{kg}^{-1}$ dose of PPR. PLA and PPR were dissolved in $0.5 \%$ sodium carboxymethyl cellulose aqueous solution to obtain a final concentration of $10 \mathrm{mg} \mathrm{mL} \mathrm{md}^{-1}$ and $5 \mathrm{mg} \mathrm{mL} \mathrm{m}^{-1}$, respectively. At various time points of $0.083,0.25,0.75,0.5,1,2,4,6,8$, 12 and $24 \mathrm{~h}$ after dosing, about $200 \mu \mathrm{L}$ of blood samples were collected from jugular vein and put into heparinized eppendorf tubes, and then centrifuged immediately at $5000 \mathrm{~g}$ for $10 \mathrm{~min}$. The supernatants were separated and used for analysis. Pharmacokinetic parameters including half-life $\left(t_{1 / 2}\right)$, maximum plasma concentration $\left(C_{\max }\right)$, time to $C_{\max }\left(T_{\max }\right)$, area under the concentration-time curve $\left(\mathrm{AUC}_{\text {last }}\right.$ and $\left.\mathrm{AUC}_{\mathrm{Inf}}\right)$, and mean residence time (MRT) of the five compounds were analyzed with DAS Version 2.0 (BioGuider Co., Shanghai, China).

\section{Measurement of SOD}

The SOD activities in supernatant plasma samples were determined by measuring the absorbance value at $450 \mathrm{~nm}$ using the SOD assay kit according to the manufacturers' instructions.

\section{Results and discussion}

\section{Optimization of chromatographic and mass spectrometry} conditions

The chromatographic behaviors and ionization responses of PPR, PPL, DPPL, PPRA, PLTR and IS were evaluated by screening several commercial reversed phase HPLC columns and different mobile phases. Terfenadine which was easily available was selected as the IS. A Phenomenex Gemini C18 column $(50 \mathrm{~mm}$ $\times 2.00 \mathrm{~mm}, 5 \mu \mathrm{m}$ ) was chosen for the separation of five analytes and IS. A gradient mobile phase system consisting of $0.1 \%$
A

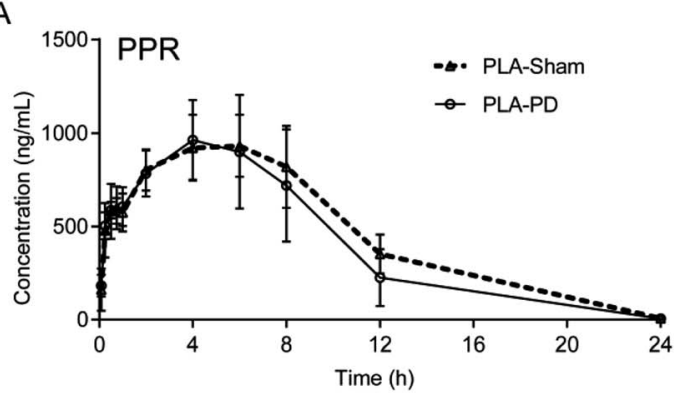

B

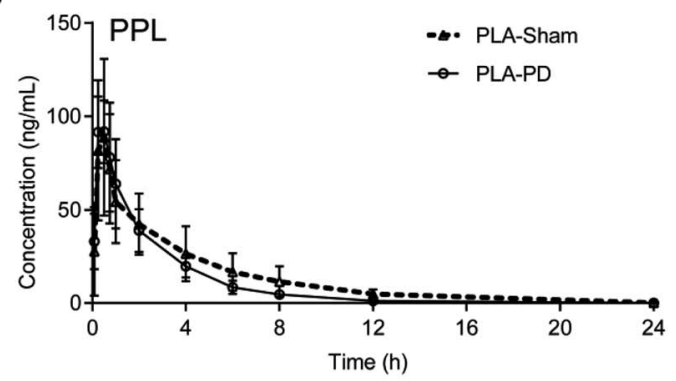

C

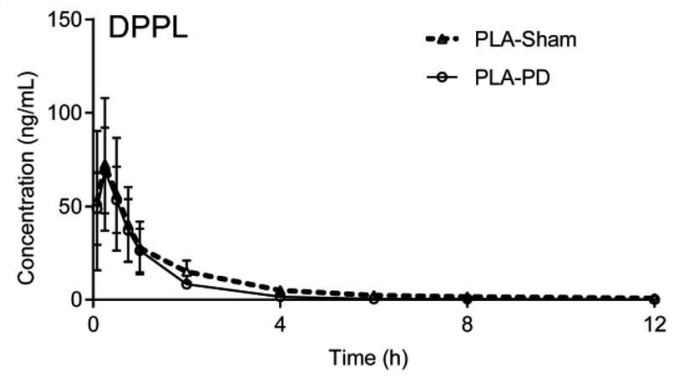

D

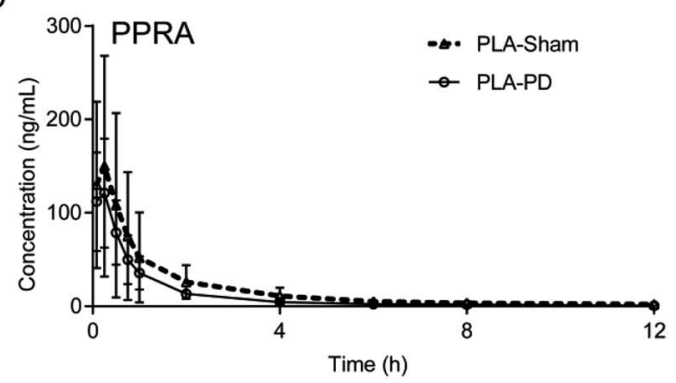

E

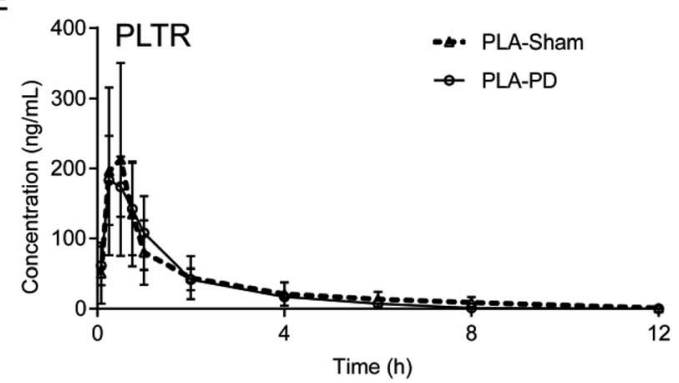

Fig. 3 The mean plasma concentration-time profiles of (A) PPR, (B) PPL, (C) DPPL, (D) PPRA, (E) PLTR in PLA-Sham and PLA-PD rats $(n=5)$.

formic acid aqueous solution (A) and acetonitrile (B) was used to obtain the acceptable peak shape and short analytical run time (4.5 minutes). The retention times were 2.51, 2.48, 2.47, 
2.49, 2.62 and $2.19 \mathrm{~min}$ for PPR, PPL, DPPL, PPRA, PLTR and terfenadine, respectively.

Due to the presence of alkaline amide group in the structures of the five analytes, they produced strong signals in ESI positive ion mode. The instrument parameters were further optimized to increase ionization efficiency. The capillary temperature was set at $550{ }^{\circ} \mathrm{C}$, and the ion spray voltage was set at $5000 \mathrm{~V}$. The optimal collision energies were set at 28, 25, 32, 38, 27 and $50 \mathrm{eV}$, respectively. Full-scan product ion spectra of $[\mathrm{M}+\mathrm{H}]^{+}$ ions and fragmentation pathways for PPR, PPL, DPPL, PPRA, PLTR and terfenadine are shown in Fig. 1. The piperidine ring of PPR and PPRA $(\mathrm{m} / \mathrm{z}$ 85), isopropylamine of PPL, DPPL and PLTR $(m / z 73)$ was broken after collision. However, the product ions including methylenedioxybenzyl $(\mathrm{m} / \mathrm{z} 135)$ of DPPL and PPRA, and $2 E, 4 E$-decadienamide $(\mathrm{m} / \mathrm{z} 168)$ of PLTR had the stronger signals. Therefore, the precursor-to-product ion transitions were monitored at $\mathrm{m} / \mathrm{z} 286.2 \rightarrow 201.2$ for PPR, $\mathrm{m} / \mathrm{z} 274.2$ $\rightarrow 201.2$ for PPL, $m / z 276.2 \rightarrow 135.1$ for DPPL, $m / z 288.2 \rightarrow$ 135.1 for PPRA, $m / z 224.1 \rightarrow 168.2$ for PLTR, and $m / z 472.1 \rightarrow$ 436.1 for terfenadine.

In general, recovery and matrix effect are significant items for the quantification of biological samples, so it is necessary to select an appropriate method of sample preparation. Liquid-liquid extraction was difficult to overall consider for these five compounds and time-consuming. And solid phase extraction was not only time-consuming, but also relatively expensive and difficult in the choice of stationary phase. In the present research, the protein precipitation method was used because of its easy operability and universal applicability, in which methanol/acetonitrile $(1: 1, \mathrm{v} / \mathrm{v})$ was used as the precipitant.

\section{Method validation}

Selectivity. Selectivity was evaluated by comparing the chromatograms of blank rat plasma, blank rat plasma spiked with standards and PLA-PD rat plasma samples. The representative chromatograms are shown in Fig. 2. No endogenous interference was observed among the five analytes and IS, their MRM indicated that they had high selectivity.
Linearity of calibration curves. The standard calibration curves were set up by weighted linear regression of the peak area ratio (y) versus concentration of each analyte $(x)$. The regression equations, correlation coefficients $(R)$, linearity ranges and LLOQs of PPR, PPL, DPPL, PPRA and PLTR are listed in Table 2. The calibration curves for five analytes showed good linearity $(R$ $>0.995)$.

Precision and accuracy. The intra/inter-day precisions and accuracies of the five alkaloids in QC samples at three concentration levels (low, medium and high) were evaluated. The results are shown in Table 3. The precisions of the high- and medium-levels QC samples were within 15\%, and that of the low-level were within $20 \%$ (RSD). The accuracies were ranged from $80 \%$ to $120 \%$. The acceptable results indicated that the established method was reliable, accurate and reproducible.

Recovery and matrix effect. As shown in Table 3, the recoveries of the five analytes were within the range of $81.10-103.72 \%$ and the matrix effects were controlled from $90.63 \%$ to $104.52 \%$. The protein precipitation method was effective, stable and free from the matrix effect.

Stability. The stabilities of 5 compounds in rat plasma under various storage and processing conditions (three freeze-thaw cycles; $-80{ }^{\circ} \mathrm{C}, 30 \mathrm{~d} ; 4{ }^{\circ} \mathrm{C}, 48 \mathrm{~h} ; 20{ }^{\circ} \mathrm{C}, 4 \mathrm{~h}$ ) were summarized in Table 4 . The RE values were less than $15 \%$, which showed that the processed samples were stable.

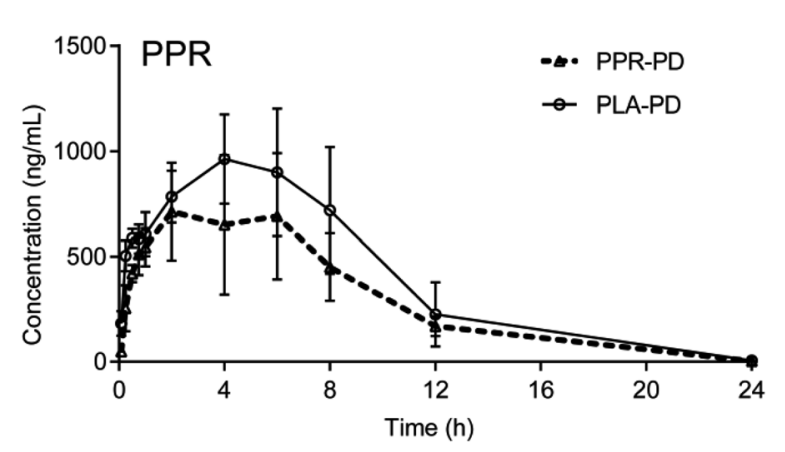

Fig. 4 The mean plasma concentration-time profiles of PPR in PLAPD and PPR-PD rats $(n=5)$.

Table 5 Pharmacokinetic parameters of PPR, PPL, DPPL, PPRA and PLTR in PLA-Sham, PLA-PD and PPR-PD rats (mean \pm SD, $n=5)$

\begin{tabular}{|c|c|c|c|c|c|c|c|}
\hline Compound & Group & $t_{1 / 2}(\mathrm{~h})$ & $T_{\max }(\mathrm{h})$ & $C_{\max }\left(\mathrm{ng} \mathrm{mL}^{-1}\right)$ & $\mathrm{AUC}_{0-t}\left(\mathrm{~h} \mathrm{ng} \mathrm{mL}^{-1}\right)$ & $\operatorname{AUC}_{0-\infty}\left(\mathrm{h} \mathrm{ng} \mathrm{mL} L^{-1}\right)$ & MRT (h) \\
\hline \multirow[t]{2}{*}{ PPR } & PLA-PD & $3.65 \pm 3.45$ & $4.00 \pm 1.41$ & $978.00 \pm 214.20$ & $9698.84 \pm 2671.21$ & $10249.90 \pm 3076.11$ & $5.99 \pm 0.63^{a}$ \\
\hline & PLA-Sham & $3.40 \pm 2.15$ & $4.80 \pm 2.28$ & $1003.20 \pm 145.58$ & $11013.21 \pm 1459.83$ & $11306.41 \pm 1519.68$ & $6.83 \pm 0.44$ \\
\hline \multirow[t]{2}{*}{ PPL } & PLA-PD & $2.42 \pm 1.23$ & $0.45 \pm 0.21$ & $97.26 \pm 17.97$ & $242.50 \pm 57.23$ & $244.98 \pm 57.49$ & $2.98 \pm 0.84^{a}$ \\
\hline & PLA-Sham & $3.68 \pm 1.32$ & $0.45 \pm 0.11$ & $89.08 \pm 41.66$ & $301.74 \pm 116.14$ & $317.72 \pm 123.19$ & $4.60 \pm 1.33$ \\
\hline DPPL & PLA-PD & $2.53 \pm 2.72$ & $0.30 \pm 0.11$ & $69.28 \pm 22.71$ & $78.18 \pm 20.32$ & $79.47 \pm 18.96$ & $1.79 \pm 1.19$ \\
\hline & PLA-Sham & $4.16 \pm 1.32$ & $0.18 \pm 0.09$ & $160.50 \pm 119.68$ & $224.89 \pm 154.54$ & $230.19 \pm 150.38$ & $3.72 \pm 1.30$ \\
\hline \multirow[t]{2}{*}{ PLTR } & PLA-PD & $1.54 \pm 0.48$ & $0.30 \pm 0.11$ & $191.80 \pm 57.69$ & $297.58 \pm 106.77$ & $310.91 \pm 105.09$ & $1.98 \pm 0.36^{a}$ \\
\hline & PLA-Sham & $2.13 \pm 0.63$ & $0.45 \pm 0.21$ & $227.40 \pm 144.69$ & $337.29 \pm 222.29$ & $354.34 \pm 231.38$ & $2.78 \pm 0.56$ \\
\hline
\end{tabular}

${ }^{a} P<0.05$ vs. PLA-Sham group. 
A

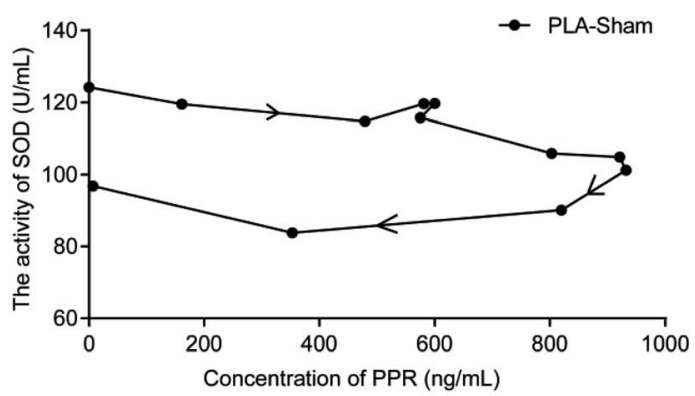

B

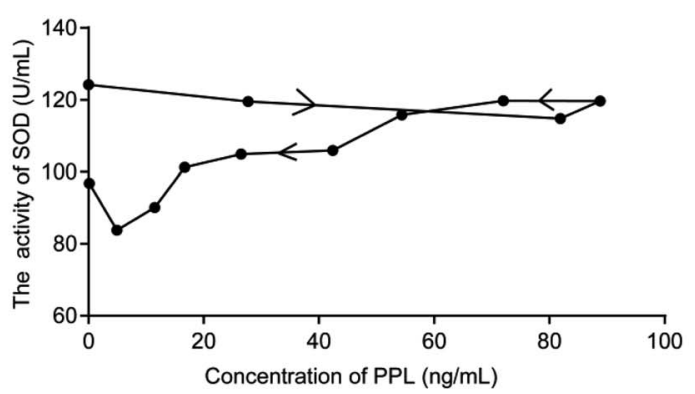

C

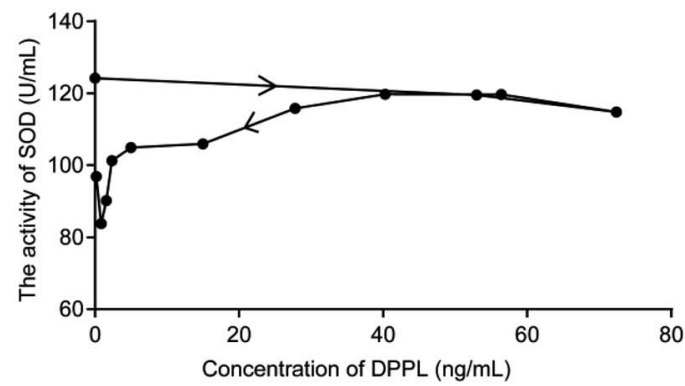

D

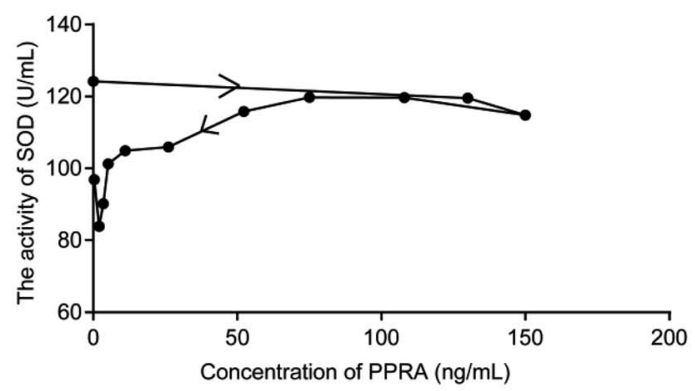

$\mathrm{E}$

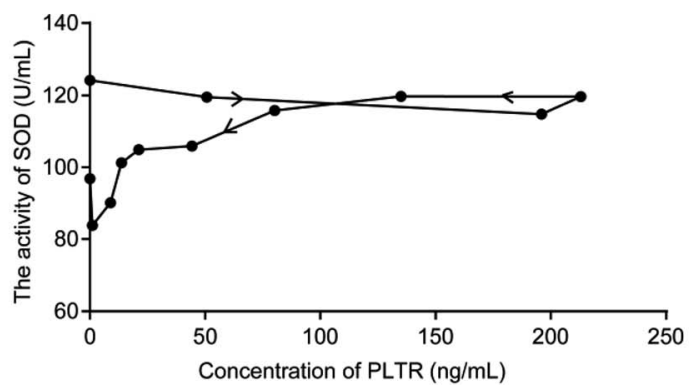

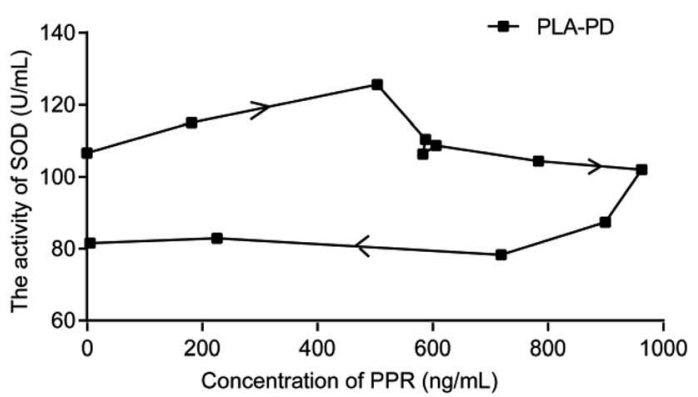
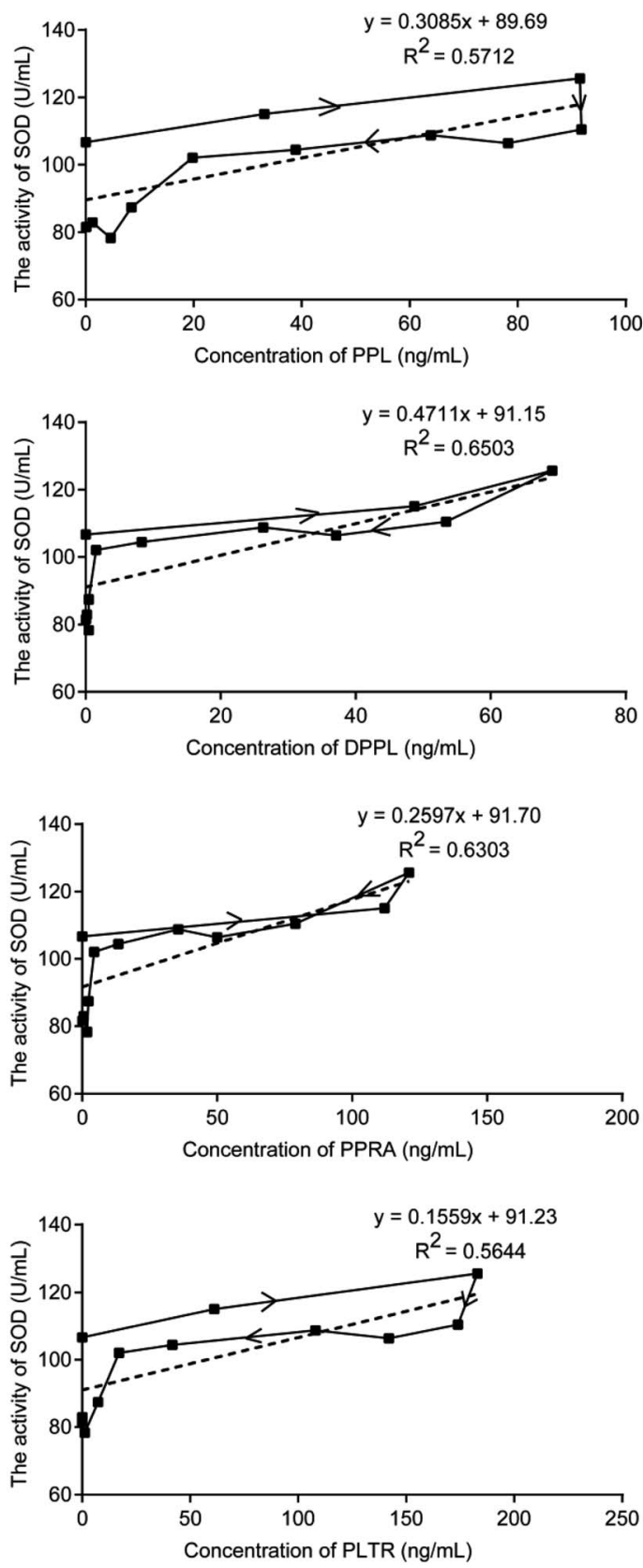

Fig. 5 Pharmacokinetic-pharmacodynamics correlation, the SOD activities in the plasma of PLA-Sham and PLA-PD rats were plotted against the plasma concentrations of (A) PPR, (B) PPL, (C) DPPL, (D) PPRA, (E) PLTR according to the different time points. (SOD activity was taken as pharmacodynamics measure). 


\section{Pharmacokinetic study}

The numbers of apomorphine-induced rotation in $30 \mathrm{~min}$ of PLA-PD group $(152 \pm 36)$ and PPR-PD group $(132 \pm 16)$ were significantly up-regulated compared with that of PLA-Sham group ( $4 \pm 1, P<0.05)$, which indicated that PD model was successfully established.

The validated UFLC-ESI-MS/MS method was successfully applied to the pharmacokinetic study of the five alkaloids in PLA-Sham and PLA-PD rats. The mean plasma concentrationtime profiles of PPR, PPL, DPPL, PPRA and PLTR are shown in Fig. 3. The main pharmacokinetic parameters are listed in Table 5. The concentrations of the five alkaloids in PLA-Sham rats were slightly higher than those in PLA-PD rats at the most time points. Compared with the corresponding pharmacokinetic parameters in PLA-Sham group, the values of $\mathrm{AUC}_{0-t}$, $\mathrm{AUC}_{0-\infty}$ and MRT for five alkaloids were slightly lower, $t_{1 / 2}$ values for PPL, DPPL, PPRA and PLTR were slightly lower, and $C_{\text {max }}$ values for PPR, DPPL, PPRA and PLTR were slightly lower. No significant differences were observed in the pharmacokinetic parameters between PLA-PD group and PLA-Sham group, except for MRTs for PPR, PPL and PLTR. In addition, the concentrations of PPR in PLA-PD rats were slightly higher than those in PPR-PD rats at the most time points, while the pharmacokinetic parameters of PPR exhibited no significant differences.

It was reported that the bioavailabilities of some drugs were significantly decreased in model rats compared with the sham rats, such as the decreased bioavailabilities of panax notoginsenosides and senkyunolide I in acute myocardial ischemia rats and biliary drainage rats, respectively, which weakened the efficacy. These results suggested that it was necessary to combine other drugs to increase the absorption of the drug to improve the therapeutic effect. ${ }^{24,25}$ However, our study revealed that there were no obvious differences in the pharmacokinetic parameters of PPR, PPL, DPPL, PPRA and PPRA between PLA-PD group and PLA-Sham group. In addition, the absorption of PPR, the active ingredient with the highest content in $P$. longum, was not obviously inhibited by its structural analogues (Fig. 4).

\section{The activity of SOD and pharmacokinetic-pharmacodynamics correlation}

Compared with the sham group $\left(124.18 \pm 9.61 \mathrm{U} \mathrm{mL}^{-1}\right)$, the basal SOD activities in the plasma of PD rats were significantly reduced $\left(106.63 \pm 11.08 \mathrm{U} \mathrm{mL}^{-1}\right)(P<0.05)$. The SOD in the plasma of PLA-PD rats reached the strongest activity at $15 \mathrm{~min}$ after orally administered PLA. The rise in SOD activity was approximately $18 \%$ as compared to the basal SOD activity. The activity of SOD was plotted against plasma concentrations of PPL, DPPL, PPRA and PLTR measured at the corresponding time points with equations of the best-fit lines $y=0.3085 x+$ $89.69\left(R^{2}=0.5712\right), y=0.4711 x+91.15\left(R^{2}=0.6503\right), y=$ $0.2597 x+91.70\left(R^{2}=0.6303\right), y=0.1559 x+91.23\left(R^{2}=0.5644\right)$, respectively (Fig. 5).

The neuroprotective effect of PLA in the PD rats was associated with antioxidation. ${ }^{6,7}$ The results showed that the SOD activities increased with the same trends of concentrations of five compounds in PD rat plasma. Especially the SOD showed the strongest activity when the concentrations of PPL, DPPL, PPRA and PLTR were at peaks in PD rat plasma. However, these were not clearly observed in the sham group. From this point of view, the antioxidant activities of PPR and its analogues were also confirmed.

\section{Conclusion}

In this study, a rapid, sensitive and selective UFLC-ESI-MS/MS method was built and validated for simultaneously determining concentrations of 5 amide alkaloids in rat plasma for the first time, and then successfully applied to the pharmacokinetic study in PD rats after oral administration of PLA. The pharmacokinetic parameters of 5 alkaloids in PD rats were hardly observed obvious differences compared with those in sham rats after oral administration of PLA except for MRTs of PPR, PPL and PLTR. The study of pharmacokinetic-pharmacodynamics correlations between SOD and concentrations of the multi-components in plasma provided some scientific information to better understand the underlying mechanism of PLA in the PD model. The results also facilitated the appropriate clinical application of PLA as a potential treatment for PD.

\section{Conflicts of interest}

There are no conflicts to declare.

\section{Acknowledgements}

This work was supported by grants from the National Natural Science Foundation of China (No. 81473333).

\section{References}

1 N. Choudhary and V. Singh, PLoS One, 2018, 13, 1-31.

2 P. Wiwattanawanichakun, A. Ratwatthananon, W. Poonsri, T. Yooboon, W. Pluempanupat, N. Piyasaengthong, S. Nobsathian and V. Bullangpoti, J. Med. Entomol., 2018, 55, 1231-1236.

3 Q. M. Ngo, P. T. Tran, M. H. Tran, J. A. Kim, S. S. Rho, C. H. Lim, J. C. Kim, M. H. Woo, J. S. Choi, J. H. Lee and B. S. Min, Phytother. Res., 2017, 31, 663-670.

4 H. Wang, J. Liu, G. Gao, X. Wu, X. M. Wang and H. Yang, Brain Res., 2016, 1639, 214-227.

5 J. Liu, W. J. Liu, Y. Q. Lu, H. Tian, C. L. Duan, L. L. Lu, G. Gao, X. Wu, X. M. Wang and H. Yang, Autophagy, 2018, 14, 845861.

6 L. Zheng, H. Wang, Y. Y. Ba, H. L. Liu, M. Wang, W. W. Guo, $\mathrm{X}$. Wu and $\mathrm{H}$. Yang, China Journal of Chinese Materia Medica, 2014, 39, 1660-1665.

7 Y. Bi, P. C. Qu, Q. S. Wang, L. Zheng, H. L. Liu, R. Luo, X. Q. Chen, Y. Y. Ba, X. Wu and H. Yang, Pharm. Biol., 2015, 53, 1516-1524.

8 H. He, W. W. Guo, R. R. Xu, X. Q. Chen, N. Zhang, X. Wu and X. M. Wang, BMC Complementary Altern. Med., 2016, 16, 412422. 
9 J. Liu, M. Chen, X. Wang, Y. Wang, C. L. Duan, G. Gao, L. L. Lu, X. Wu, X. M. Wang and H. Yang, OncoTargets Ther., 2016, 7, 60823-60843.

10 C. H. Lin, K. W. Hsu, C. H. Chen, Y. S. Uang and C. J. Lin, Biochem. Pharmacol., 2017, 142, 216-228.

11 N. N. Wang, X. P. Wang, Y. Zhang, Q. Y. Zhang, P. C. Xu, H. L. Xin, R. J. Wu, D. Shou and L. P. Qin, J. Pharm. Biomed. Anal., 2018, 154, 460-467.

12 Y. Jin, Y. P. Tang, Z. H. Zhu, E. X. Shang, H. Q. Pang, X. Q. Shi, Y. Y. Chen, J. Wang, X. Chang, A. Kang, G. S. Zhou, Y. J. Shi, J. Sun, Z. S. Tang, S. P. Li and J. A. Duan, Molecules, 2017, 22, 1746-1758.

13 C. X. Lv, Q. Li, Y. W. Zhang, Z. Y. Sui, B. S. He, H. R. Xu, Y. D. Yin, X. H. Chen and K. S. Bi, J. Mass Spectrom., 2013, 48, 904-913.

14 W. B. Xie, X. J. Qiu, X. Huang, Y. Xie, K. G. Wu, Y. Wang, H. Ji, J. He and P. Ren, Exp. Ther. Med., 2013, 6, 913-918.

15 B. S. He, Q. Li, Y. Jia, L. S. Zhao, F. Xiao, C. X. Lv, H. R. Xu, X. H. Chen and K. S. Bi, J. Mass Spectrom., 2012, 47, 13331340.

16 Y. H. Wang, Y. L. Hong, Y. Feng, D. S. Xu, S. Liang, X. Lin and L. Shen, Eur. J. Drug Metab. Pharmacokinet., 2012, 37, 91-97. 17 F. L. Pagan, M. L. Hebron, B. Wilmarth, Y. Torres-Yaghi, A. Lawler, E. E. Mundel, N. Yusuf, N. J. Starr, J. Arellano,
H. H. Howard, M. Peyton, S. Matar, X. Liu, A. J. Fowler, S. L. Schwartz, J. Ahn and C. Moussa, Pharmacol. Res. Perspect., 2019, 7, e00470.

18 F. Stocchi, L. Vacca, P. Stirpe and M. Torti, Expert Opin. Drug Metab. Toxicol., 2018, 14, 1189-1195.

19 T. Müller and J. D. Möhr, Expert Opin. Pharmacother., 2018, 19, 2063-2071.

20 M. Nomoto, M. Nagai, N. Nishikawa, R. Ando, Y. Kagamiishi, K. Yano, S. Saito and A. Takeda, eNeurologicalSci, 2018, 13, 813.

21 X. F. Sun, D. Q. Wang, Z. E. Wu, P. Li, J. Z. Niu, J. F. Wang and W. Wang, Chin. J. Exp. Tradit. Med. Formulae, 2011, 17, 111115.

22 J. H. Liu, Y. Bi, R. Luo and X. Wu, J. Chromatogr. B: Anal. Technol. Biomed. Life Sci., 2011, 879, 2885-2890.

23 H. L. Liu, R. Luo, X. Q. Chen, J. H. Liu, Y. Bi, L. Zheng and X. Wu, J. Chromatogr. B: Anal. Technol. Biomed. Life Sci., 2013, 928, 78-82.

24 H. J. Zhi, Y. P. Deng, B. P. Yan, Z. W. Li, S. M. Han, Y. B. Zhang, J. J. Hou, W. Y. Wu and D. Guo, J. Pharm. Biomed. Anal., 2019, 166, 52-65.

25 H. G. Li, Y. Jiang, Y. Wang, H. Y. Lv, H. T. Xie, G. P. Yang, C. X. Guo, J. Tang and T. Tang, Front. Pharmacol., 2018, 9, 1461-1468. 